\title{
A quantitative study of auditory-evoked saccadic eye movements in two dimensions
}

Received: 8 February 1995/ Accepted: 12 June 1995

\begin{abstract}
We investigated the properties of human saccadic eye movements evoked by acoustic stimuli in the two-dimensional frontal plane. These movements proved to be quite accurate, both in azimuth and in elevation, provided the sound source spectrum had a broad bandwidth and a sufficiently long duration. If the acoustic target was a tone, the azimuth of the saccadic end points remained equally accurate, whereas the elevation of the response was related to the frequency of the tone, rather than to the physical position of the target. Saccade elevation accuracy also declined substantially for short-duration noise bursts, although response elevation remained highly correlated with target elevation. The latencies of auditory saccades depended on the amplitude, but not on the direction of the eye movement, suggesting a polar coordinate origin of auditory saccade initiation. We also observed that the trajectories of auditory saccades were often substantially curved. Both a qualitative and a model-based analysis showed that this curvature corrected for errors in the initial direction of the saccade. The latter analysis also suggested that the kinematic properties of auditory saccades could be described by the superposition of two overlapping saccadic eye movements, hypothesized to be based on binaural difference cues and monaural spectral cues in the auditory signal, respectively. It is argued that, although the audio-oculomotor system has to operate in a feedforward way, it must nevertheless have access to an accurate representation of actual and desired eye position. Different models underlying the generation of auditory saccades are discussed.
\end{abstract}

\footnotetext{
M. A. Frens ()$^{1}$ - A. J. Van Opstal

Department of Medical Physics and Biophysics,

University of Nijmegen, P.O. Box 9101 ,

$6500 \mathrm{HB}$ Nijmegen, The Netherlands;

Fax: +31-80-541435; e-mail: maarten@mbfys.kun.nl

Present address:

1 Vestibular Laboratory, Neurology Department, University Hospital, CH-8091 Zürich, Switzerland;

Tel.: +41-1-2555592; Fax: +41-1-2554507;

e-mail: mfrens@neurol.unizh.ch
}

Key words Auditory localization - Saccades - Model . Listing's law - Human

\section{Introduction}

In this paper, auditory-evoked saccadic eye movements (auditory saccades) are investigated in two dimensions. Specifically, the influence of the acoustic spectrum on saccade accuracy is studied. In addition, we will discuss how the kinematic properties of auditory saccades may shed more light on the way in which these movements are programmed by the audio-oculomotor pathway. We have studied human saccadic eye movement responses towards auditory stimuli for two main reasons. First, there exist several nontrivial differences between the representation of targets by the auditory and visual systems, which make the auditory system an interesting tool for gaining new insights into the programming of saccades. Secondly, auditory saccades may serve as a precise, fast, and natural pointer for assessing the processes underlying auditory localization. Up to now, the great majority of studies using eye movements for probing auditory localization have been limited to the horizontal plane (e.g. Zahn et al. 1979; Whittington et al. 1981; Zambarbieri et al. 1981, 1982; Lueck et al. 1990). To our knowledge, the number of studies involving auditory saccades over the full oculomotor range in two dimensions is still very limited (Jay and Sparks 1990; Frens and Van Opstal 1994a,b). However, as will be argued below, the extension from the horizontal domain to acoustic stimuli presented over the full two-dimensional oculomotor range is an essential one, involving a number of nontrivial additional problems.

Unlike the visual or somatosensory systems, the auditory system has no topographically organized representation of target position at the level of its sense organs. Whereas the position of a visual stimulus relative to the fovea corresponds in a one-to-one fashion to the locus of activity on the retina, cochlear hair cells are narrowly tuned to auditory stimuli of a specific frequency, irre- 
spective of stimulus location. Due to the mechanical properties of the basilar membrane, the acoustic information is tonotopically organized, resulting in a spectral code for the auditory system. This tonotopic organization is preserved in the majority of subcortical as well as cortical auditory centres (e.g. Clarey et al. 1992; Irvine 1992, for an extensive review).

As a consequence, spatially accurate auditory saccades have to be programmed on the basis of implicit cues in this acoustic spectral representation (see below). It is therefore conceivable that the spectral content of an auditory stimulus may play a role in determining the evoked saccadic response.

A spatial representation of the acoustic world has to be derived by the auditory system from monaural as well as from binaural acoustic cues. It is thus recognized that the horizontal component of the auditory stimulus position (target azimuth) is extracted on the basis of different cues - and, presumably, through a different neural pathway - from the vertical component (target elevation).

Target azimuth is predominantly determined by interaural timing and intensity differences (e.g. Middlebrooks and Green 1991). Since in humans the pinnae are symmetrically positioned on the head, no changes in binaural differences result for sound position changes in the vertical direction. However, due to the direction-dependent spectral filtering properties of the pinnae, target elevation can be derived on the basis of the resulting monaural spectral cues (e.g. Middlebrooks and Green 1991; Kistler and Wightman 1992), even when knowledge of the original, unfiltered, sound spectrum is not available (Zakarouskas and Cynader 1993).

As a result, the auditory localization process is initially performed in a Cartesian coordinate system, since separate neural pathways underlie the horizontal and the vertical component of the auditory spatial percept. By contrast, retinotopic maps in the visual system seem to be organized in polar coordinates, reflecting the radial symmetry of the retina.

Since in humans the pinnae are immobile with respect to the head, the acoustic cues provide the auditory sytem with a code expressed in a craniocentric frame of reference. Note that this feature constitutes an interesting additional difference from the well-established oculocentric organization of the visuomotor system.

A further difference between saccades evoked by auditory and visual stimuli, resides in the fact that, in the absence of head movements, the former cannot provide the system with sensory feedback during or after the eye movement. It is thus expected that, in the absence of visual cues, the audio-oculomotor system is not able to evaluate the accuracy of the targeting saccade. We will come back to this point in the Results.

\section{Previous studies}

Most studies involving auditory saccades have been confined to stimuli and movements in the horizontal plane.
In what follows, we will briefly review the available literature on the properties of these responses, as far as their timing, accuracy and kinematics are concerned.

\section{Response latency}

There appears to be a tendency for the latency of horizontal auditory saccades to decrease with target eccentricity (Zahn et al. 1979; Zambarbieri et al. 1981). Remarkably, the factor that determines saccade latency is the eccentricity of the target not with respect to the head, but rather relative to the fovea. For example, shifting the position of the initial visual fixation spot to a different (horizontal) position, while keeping the acoustic target stimulus fixed with respect to the head, leads to a decrease in the saccade reaction time (Zahn et al. 1979; Jay and Sparks 1990). By contrast, the latency of saccades to visual stimuli has the tendency to increase with target eccentricity relative to the fovea (Kalesnykas and Hallett 1994).

\section{Response accuracy}

Though inferior to saccadic responses towards visual targets, the accuracy of auditory saccades can be quite high. To our knowledge, the only study which has investigated saccade accuracy for randomly presented auditory targets in two dimensions is by Jay and Sparks (1990), who report a "mean error" of about $6^{\circ}$ for humans. It is not clear, however, whether this error was due to scatter in the saccade endpoints or to systematic mislocalizations of the targets. These authors also report that auditory saccades towards horizontal targets are slightly more accurate than saccades towards targets in the vertical direction.

Both Lueck et al. (1990) and Zambarbieri et al. (1981) report a first-saccadic response amplitude which is only about $65 \%$ of the acoustic target eccentricity. Although these data suggest a much poorer performance of the audio-oculomotor system than the data of Jay and Sparks (1990), a potentially important difference between these studies resides in the applied sounds. Whereas in the latter study broad-band noise stimuli were presented to the subjects, the former researchers used stimuli with a much more limited amplitude spectrum. As described above, the sound spectrum may be expected to exert an influence on the auditory-evoked orienting response.

Studies with macaque monkeys have shown that the accuracy of auditory saccades to targets on the horizontal meridian does not depend on the starting position of the eyes (Whittington et al. 1981). Recordings in the deep layers of the monkey superior colliculus (SC) suggest that the necessary coordinate transformation from the initially craniocentric auditory code (see above) into an appropriate oculocentric motor command is already complete at this level (Jay and Sparks 1987). 


\section{Saccade kinematics}

Visual saccades obey stereotyped amplitude-duration as well as amplitude-peak velocity relationships, which have become known as the "main sequence" for saccades (Bahill et al. 1975). More recently, these relations have been shown to retain their characteristics for saccade vectors in two dimensions (Van Gisbergen et al. 1985).

In two dimensions, the trajectories of human visual saccades are approximately straight (e.g. Fig. 6). It has been recognized that this property reflects important aspects of the neural organization of the visuomotor pathway, because it implies that throughout the saccade the horizontal and vertical components of the eye movement must be scaled versions of each other and hence tightly coupled (see Van Gisbergen et al. 1985, for a theoretical analysis). In a later experimental study, it was demonstrated that this argument could also be applied for saccades towards remembered visual targets, even though such movements are generally slower than visually guided movements (Smit et al. 1990).

It has been reported repeatedly that horizontal eye movements directed at acoustic targets are slower and more variable than visually evoked saccades of the same amplitude, in the sense that their peak velocity is lower and their movement duration is longer (Zambarbieri et al. 1981, 1982; Jay and Sparks 1990).

As will be demonstrated below (see Results), also in two dimensions, auditory saccades are less stereotyped than their visual counterparts. First, their spatial trajectories are more curved than visually evoked saccade traces, suggesting a weaker coupling between the horizontal and vertical velocity channels. Second, the velocity profiles are endowed with more variation. Because the auditory target position appears to be initially encoded in Cartesian coordinates through separate neural pathways (azimuth-elevation; see above), we wondered whether this property would perhaps be reflected in the trajectories and kinematics of auditory saccades. To that means, we have developed a decomposition procedure in order to further analyse these movements.

A preliminary account of the data in this study has been given in Frens and Van Opstal 1994a,b.

\section{Materials and methods}

\section{Experimental setup}

Experiments were performed in a completely dark, sound-attenuated room $(3 \times 3 \times 3 \mathrm{~m})$, in which walls, ceiling and floor, as well as large objects, had been covered with acoustic foam that prevented echoes of sound frequencies above $500 \mathrm{~Hz}$. The mean background noise level was $35 \mathrm{~dB}$ (SPL).

\section{Subjects}

Subjects were one female and five male human volunteers (21-37 years old). All subjects were without any known uncorrected visual, auditory or motor disorder, with the exception of J.O., who was amblyopic in his right eye. During the experiment, sub- jects were comfortably seated in a chair; head movements were restrained by a soft support at the back of the subject's head. Viewing was binocular. A sixth subject (A.M.) participated in the recording of auditory saccades in three dimensions (see also below).

\section{Auditory stimuli}

Sound stimuli were delivered through a speaker (Philips AD44725; radius $43 \mathrm{~mm}$ ) that was mounted on a two-joint robot arm, equipped with stepping motors (type VRDM5; Berger Lahr). This robot arm enabled rapid positioning of the speaker anywhere on the surface of a virtual sphere with a radius of $90 \mathrm{~cm}$ and its centre at the subject's head.

Auditory noise stimuli of $500 \mathrm{~ms}$ duration consisted of bandpass-filtered white noise $(150 \mathrm{~Hz}-20 \mathrm{kHz}$; Krohn-Hite 3343$)$, generated by a noise-generator (Hewlett-Packard HOI-3722a). The slight deviations of the speaker's frequency response from a flat amplitude spectrum were not corrected for. Tone stimuli $(0.5,1.0$, $2.0,5.0$ and $10 \mathrm{kHz}$ ) and short-duration noise bursts (see below) were generated by a PC-80486, equipped with a digital-analogue converter (Data Translation 2821 board). The first $5 \mathrm{~ms}$ and the last $5 \mathrm{~ms}$ of the tone signals were smoothed with a sine-shaped filter, which masked sudden on- and offsets of the stimulus. The noise bursts had a 1-ms rise and fall time. All sound stimuli were amplified (Luxman 58A) to about $60 \mathrm{~dB}$ SPL at the position of the subject's head.

\section{Visual stimuli}

Visual targets were red light-emitting diodes (LEDs; radius $2.5 \mathrm{~mm}$, subtending $0.2^{\circ}$ as viewed from the subject), mounted on an acoustically transparent wire frame that constituted a halfsphere just proximal to the working range of the robot. The distance between the LEDs and the subject was $85 \mathrm{~cm}$; the applied intensity was $0.15 \mathrm{~cd} \cdot \mathrm{m}^{-2}$.

\section{Measurements}

The horizontal and vertical components of the position of the right eye were measured by means of the scleral coil technique (Collewijn et al. 1975). Two sets of horizontal and vertical coils, attached along the edges of the room, generated the magnetic fields. In this way, the measuring apparatus caused no acoustic reflections. The spatial resolution of this method was better than $0.5^{\circ}$ over the entire oculomotor range.

In one experimental session we recorded eye movements in three dimensions, by applying the dual scleral-coil technique described by Collewijn et al. (1985). Details of the method for absolute calibration of this eye-coil system are fully described by Hess et al. (1992).

Data acquisition and the timing of the stimulus events were both controlled by a PC-80386, equipped with a data-acquisition board (Metrabyte DAS16) and a digital I/O card (Data Translation 2817). This computer communicated through its parallel port with the PC-80486 that controlled the robot with the speaker. The sampling rate was $500 \mathrm{~Hz}$ for each eye position channel. Each trial consisted of $2 \mathrm{~s}$ of recording time, starting $400 \mathrm{~ms}$ before presentation of the peripheral stimuli.

\section{Experimental protocol}

Subjects were instructed to fixate an initial LED at the straightahead position. After a random period of $1-2.5 \mathrm{~s}$, this fixation spot extinguished and, simultaneously, a randomly selected peripheral target was presented. The default target duration was $500 \mathrm{~ms}$. In a separate set of experiments (subjects J.O., P.H. and J.G. only) we also applied noise-burst durations of 3,5 , and $10 \mathrm{~ms}$, respectively. Subjects had to redirect their eyes as quickly and as accurately as 
possible towards the new stimulus. A typical experimental session consisted of the following paradigms: (1) one experimental set of visual stimuli; (2) one or several sets of auditory broad-band noise stimuli; (3) one or several sets of tone stimuli of various frequencies.

Visual target positions were at spherical polar coordinates $R \in[2,5,9,14,20,27,35]^{\circ}$ and $\Phi \in[0,30,60 \ldots 330]^{\circ}$, where $\Phi=0^{\circ}$ corresponds to a rightward position and $\Phi=90^{\circ}$ is upward. $R$ is the distance from the initial fixation spot. Total number of trials: $n=84$.

Auditory target positions (either noise bursts or tones) were presented at $R \in[10,20,30]^{\circ}$ and $\Phi \in[0,45,90 \ldots 315]^{\circ}$. In a given set, each position was presented three times $(n=72)$.

During auditory experiments the speaker was moved, between trials and in complete darkness, to a random position and subsequently to the randomly selected target position. This procedure denied the subject both visual and auditory cues regarding the new stimulus position. All subjects reported the impossibility of identifying the stimulus location on the basis of sounds produced by the stepping motors. This was confirmed in a control experiment with two of our subjects. In this experiment, identical stimulus movement procedures were followed, but no sound stimulus was delivered. The subject was asked to redirect their gaze towards the "guessed" stimulus position as soon as the central fixation LED was extinguished. The correlation coefficients between the subjects' first-saccade vectors and horizontal and vertical speaker position was insignificant [subject J.O.: $r_{\mathrm{H}}=-0.13, r_{\mathrm{V}}=-0.13$; subject P.H.: $\left.r_{\mathrm{H}}=0.02, r_{\mathrm{V}}=0.01 ; n=25, P(r)>0.2\right]$. Thus, these control experiments clearly showed that no additional cues underlay the subjects' performance in the audio-oculomotor task. This conclusion is further supported by the tone and short-duration noise-burst experiments (see Results).

\section{Data analysis}

Eye position signals were calibrated off line on the basis of final fixation positions obtained in the visual test set. Raw data signals were calibrated by applying a back propagation algorithm (e.g. Rumelhart et al. 1986). This algorithm could cope with small inhomogeneities in the magnetic fields as well as with minor crosstalk components between the two eye movement channels.

Since all targets were at the same distance from the subject, the azimuth, $A$, and elevation, $E$, of the auditory target position are related to the spherical polar angles $(R, \Phi)$ by:

$A=\arcsin (\sin R \cdot \cos \Phi) \approx R \cdot \cos \Phi$

$E=\arcsin (\sin R \cdot \sin \Phi) \approx R \cdot \sin \Phi$

Both the $(A, E)$ and the $(R, \Phi)$ coordinate systems have their origin at the subject's straight-ahead fixation direction. The approximation, which corresponds to the horizontal and vertical target coordinates, holds within $5 \%$ for eccentricities $R<35^{\circ}$. In this way, the azimuth and elevation of target positions could be directly matched to the horizontal and vertical eye position channels.

Saccades were detected off line by a computer algorithm which applied separate eye velocity and mean acceleration criteria for onset and offset, respectively. Detection markings were visually checked by the experimenter and could be interactively changed if necessary. Movements associated with blinks were discarded from further analysis. Several parameters were extracted for each saccade: amplitude $(R)$, taken as the length of the vector between onset and offset position, direction $(\Phi)$, peak eye velocity $\left(V_{\max }\right)$, duration $(D)$, movement latency re stimulus onset $(L)$, initial saccade direction $\left(\Phi_{0}\right)$, and saccade curvature $(C)$.

The initial direction was taken as the direction of the saccade at a distance of $1^{\circ}$ from its starting point. We have checked that changing this criterion up to $5^{\circ}$ did not appreciably change the value of $\Phi_{0}$.

Saccade curvature was defined as the maximal deviation $(M)$ of the saccade trajectory from a straight line, normalized by saccade amplitude: $C= \pm M / R$ (negative value, clockwise curvature; see Smit et al. 1990 for details). For example, a trajectory having the shape of an anti-clockwise semi-circle would yield $C=+0.5$.

\section{Decomposition procedure of auditory saccades}

The two-dimensional saccade trajectory is determined by a horizontal and a vertical eye position component:

$\mathbf{T}(\mathbf{t})=[H(t), V(t)]$

In order to further study the properties of auditory saccades in two dimensions and to assess in a robust way whether the observed curvature may be interpreted as a midflight error correction (see Results), we have applied a decomposition procedure to these movements. This method aimed at reconstructing, from the original saccade trajectory, the contribution of two straight eye movements which overlap in time. It should be noted that such a decomposition is not unique, but may be achieved by many different combinations.

In general, the decomposition assigns to the original trajectory two constituent movements: a primary movement, $\mathbf{P}(t)$, and a secondary movement, $\mathbf{S}(t)$ :

$\mathbf{T}(t)=\mathbf{P}(t)+\mathbf{S}(t)$

Since both movements are assumed to be straight, their horizontal and vertical components must be scaled versions of each other. Thus,

$\mathbf{P}(t)=\left[H_{P}(t), \alpha \cdot H_{P}(t)\right] \quad$ and $\quad \mathbf{S}(t)=\left[H_{S}(t), \beta \cdot H_{S}(t)\right]$

It follows from Eq. 3 that the secondary movement is fully determined by the choice of $H_{p}(t)$ and $\alpha$ :

$\mathbf{S}(t)=\left[H(t)-H_{P}(t), V(t)-\alpha \cdot H_{P}(t)\right]$

However, most of these choices are not related to a conceivable motor strategy underlying the curved saccade trajectory. For reasons which will be explained in more detail in the Discussion, we have selected a particular solution for the auditory saccades (see Fig. 1B): (1) the primary movement (P-movement) has a direction equal to the initial saccade direction $\Phi_{0} ;(2)$ the secondary movement (S-movement) has a purely vertical direction. Thus, the decomposition procedure of auditory saccades amounts to the following choice:

$H_{p}(t)=H(t)$

$\alpha=\tan \left(\Phi_{0}\right)$

Note that, according to this proposal, a straight movement will always lead to a negligible secondary movement. Auditory-evoked movements were considered straight whenever the amplitude of the S-movement fell below $1^{\circ}$. Such small S-movements were not incorporated in our analysis. We have also excluded movements having a starting direction within $10^{\circ}$ of the purely vertical directions, since P- and S-movements would then have to be computed on the basis of very small, and hence unreliable, horizontal saccade components [ $\tan \left(\Phi_{0}\right)$ approaches infinity]. Note also that, according to this procedure, no assumptions are made as to the saccadic or non-saccadic nature of the constituent movements. The subsequent steps underlying the applied decomposition procedure are illustrated in Fig. 1A.

\section{Results}

\section{Accuracy}

As can be observed in Fig. 2 (data from subject M.F.), auditory saccades towards broad-band noise stimuli (centre column) were quite accurate, although somewhat inferior to the accuracy of primary saccades to visual targets (left column). Correlation coefficients between auditory target azimuth and first-saccade endpoint azimuth were highly significant, and linear regression lines had a 
Fig. 1A, B Decomposition procedure. A Horizontal (left) and vertical (right) velocity signals of a curved auditory saccade (top), from subject M.F., of the reconstructed P-movement (centre) and of the Smovement (bottom). See text for further details. B Spatial tory saccade and the computed $\mathrm{P}$ - and S-movements. Note that the P-movement has a direction equal to the initial direction of the auditory saccade; the Smovement is purely vertical by definition trajectories of the original audi-
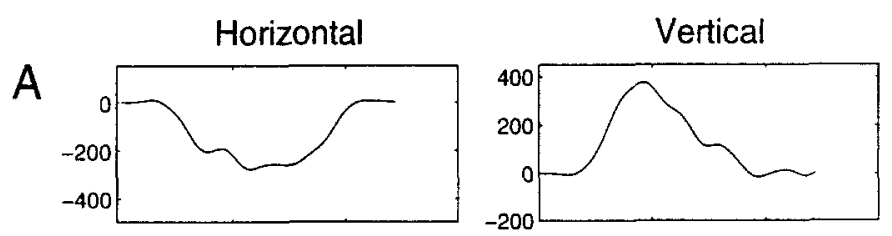

$\mathrm{T}$
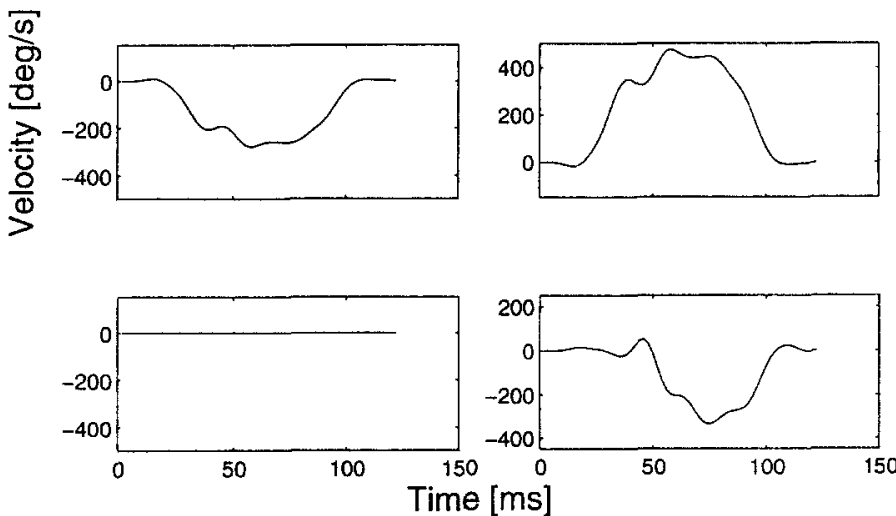

$S$

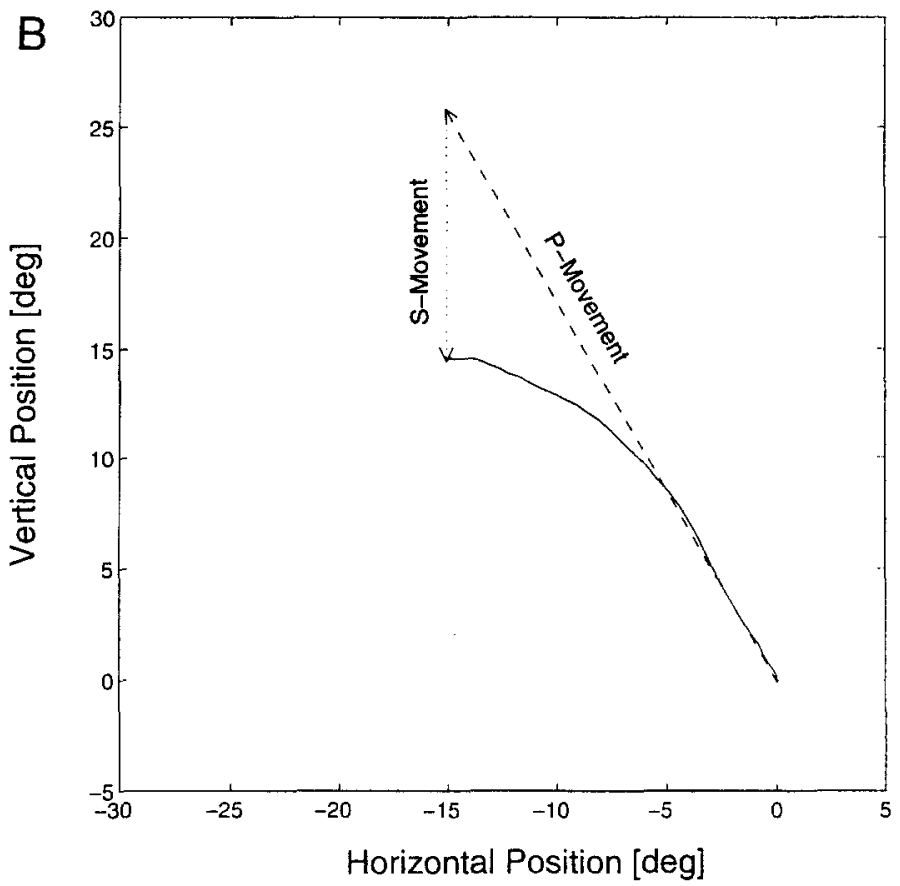

slope which was close to 1 (see Table 1 for data of all subjects). Auditory saccades were accurate in both dimensions, although, for all subjects, the correlation coefficients for the elevation component were consistently somewhat lower than for the azimuth component.

The accuracy of auditory saccades appeared to depend strongly on the spectral content of the acoustic stimulus. When tone stimuli were presented instead of broad-band noise, subjects failed to respond to the elevation component of the stimuli, resulting in insignificant correlations for the frequencies applied in this study $(r$ often less than 0.1 ).

An example is presented in Fig. 2 (right column) for the auditory-evoked saccades of subject M.F. towards tonal sounds of $500 \mathrm{~Hz}$ presented at the same locations in the two-dimensional frontal plane as the broad-band noise stimuli. Note that the azimuth component of the tone-evoked saccades (top-right panel) remained about as accurate as for auditory saccades under broad-band noise conditions (top-centre panel).

As is illustrated in Fig. 3 for three of our subjects, it appeared that saccade elevation was confined to a narrow range, centred around a frequency-dependent and idiosyncratic mean value (see Table 1 for the quantitative results for all subjects). As a result, tone saccades had an elevation component that was independent of target position. The standard deviation around the tone-specific mean value was comparable with the amount of elevation scatter obtained under broad-band noise conditions (cf. Fig. 2, bottom centre and right panels). Thus, the 
Fig. 2 Accuracy of primary saccades. End-point azimuth (top) and elevation (bottom) of primary saccades to targets in the two-dimensional frontal plane (see Materials and methods) are shown as a function of target azimuth and elevation, respectively. Data are from subject M.F. Left Visually directed saccades, centre saccades towards broad-band noise targets $(500 \mathrm{~ms})$, right saccades towards a $500-\mathrm{Hz}$ tone. The latter data are typical for all tone responses, apart from the mean value of the saccade elevation components (see also Fig. 3)
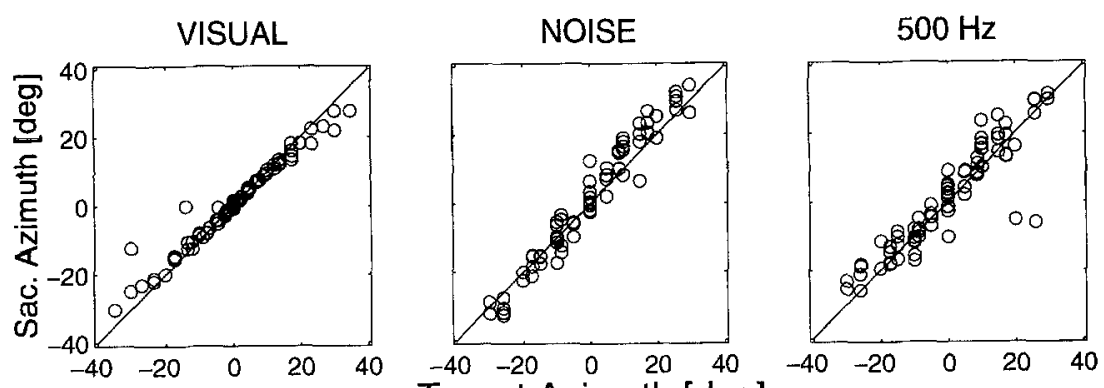

Target Azimuth [deg]
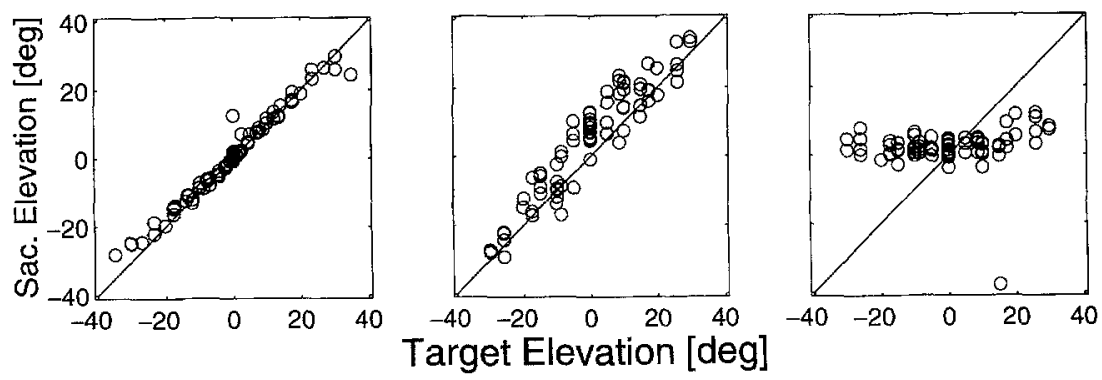

Table 1 Accuracy of horizontal and vertical components of the first visual saccades and auditory saccades towards broad-band noise and tone stimuli. Values are fitted slopes and correlation coefficients of linear regression lines between saccade and target components. Values for tone saccades are means and standard deviations, pooled for the different tones $(n=5)$. Despite the slightly higher correlations for visual saccades, broad-band noise saccades are very accurate in both components. Note that the slopes for auditory azimuth are closer to 1.0 than for visual saccades. The azimuth components of tone saccades are almost as accurate as for broad-band noise saccades. Elevation components of tone saccades, however, are not related to sound target elevation

\begin{tabular}{lllll}
\hline Subject & Visual & Noise & & Tones \\
\cline { 2 - 2 } \cline { 5 - 6 } Slope $r$ & Slope $r$ & & Slope & $r$ \\
\hline
\end{tabular}

Azimuth

$\begin{array}{lllllll}\text { JG } & 0.85 & 0.89 & 0.90 & 0.95 & 0.93 \pm 0.21 & 0.87 \pm 0.13 \\ \text { JO } & 0.83 & 0.99 & 0.83 & 0.97 & 0.79 \pm 0.04 & 0.96 \pm 0.01 \\ \text { MF } & 0.87 & 0.99 & 1.20 & 0.98 & 0.93 \pm 0.14 & 0.88 \pm 0.04 \\ \text { NC } & 0.88 & 0.99 & 0.89 & 0.81 & 0.75 \pm 0.18 & 0.92 \pm 0.05 \\ \text { PH } & 0.88 & 0.99 & 0.94 & 0.95 & 0.81 \pm 0.17 & 0.88 \pm 0.09 \\ \text { RW } & 0.80 & 0.93 & 0.95 & 0.95 & - & \end{array}$

Elevation

\begin{tabular}{lllllrr} 
JG & 0.81 & 0.91 & 0.92 & 0.91 & $0.05 \pm 0.08$ & $0.10 \pm 0.17$ \\
JO & 0.88 & 0.99 & 0.54 & 0.87 & $0.03 \pm 0.03$ & $0.15 \pm 0.15$ \\
MF & 0.88 & 0.99 & 0.96 & 0.90 & $-0.01 \pm 0.03$ & $-0.02 \pm 0.08$ \\
NC & 0.88 & 0.99 & 0.77 & 0.81 & $-0.02 \pm 0.03$ & $0.06 \pm 0.05$ \\
PH & 0.80 & 0.95 & 0.91 & 0.85 & $0.02 \pm 0.09$ & $0.08 \pm 0.28$ \\
RW & 0.82 & 0.86 & 0.72 & 0.91 & - & \\
\hline
\end{tabular}

overall endpoint distribution of tone-evoked saccades was horizontally stretched (Fig. 2, right column).

In order to assess the effect of the duration of a broadband noise stimulus on saccade accuracy, three of our subjects also participated in an experimental session in which the auditory stimulus consisted of a short-duration noise burst (3,5 and $10 \mathrm{~ms}$; the 500-ms noise burst served as a control stimulus). In Fig. 4A the four different sound pressure signals, measured at the position of the subject's head, together with the associated power spectra, are shown. As may be seen in Fig. 4B, C (data from subject P.H.; stimuli indicated by different symbols), in which the localization results are presented in the same format as in Fig. 2, the overall performance deteriorates dramatically as the stimulus duration decreases. The effect is particularly strong on the elevation component (Fig. 4C). Under all conditions tested, however, the azimuth of the first saccadic response was not statistically different from the long-duration condition (Fig. 4B). This feature was consistently obtained for all three subjects tested. This is shown in Fig. 4D, in which the slope of the regression line (target position component vs first-saccade component) has been plotted as a function of stimulus duration. Note that, for a stimulus duration of $10 \mathrm{~ms}$, elevation performance has not yet reached the quality obtained for the 500-ms stimuli. The slopes of the azimuth curves, however, already reach their optimal value for stimulus durations of less than $5 \mathrm{~ms}$. The quantitative results of these experiments have been summarized in Table 2 .

\section{Latency}

In Fig. 5 we illustrate how saccade latency and saccade amplitude are related. Figure 5A shows that the auditory reaction times (circles, solid fit curve) tend to decrease with increasing amplitude (data from J.O., pooled for all saccade directions). In order to quantify this effect, we fitted the data with the following exponential function:

$L=L_{\infty}+L_{s} \cdot e^{-\alpha R}$

where $L$ is the onset latency for the saccades in milliseconds, $R$ is saccade amplitude in degrees, and $\alpha$ (in $\mathrm{deg}^{-1}$ ), $L_{\infty}$ and $L_{s}$ (both in milliseconds) are constant parameters. For auditory saccades, the relation shown in Fig. 5A was very robust and quite typical for all six sub- 
Fig. 3 Dependence of response elevation on sound frequency. Relationship between the frequency of the tone stimulus and the mean elevation of the saccade endpoints for three different subjects. Auditory targets were presented in the two-dimensional frontal plane (see Materials and methods, and Fig. 2). Error bars denote 1 SD. Note different scales for saccade elevation. Subject M.F. predominantly made saccades with an upward component (left), subject P.H. tended to generate saccades in a downward direction (centre), whereas subject N.C. (right) made saccades over the entire oculomotor elevation range. The azimuth component of the saccades (not shown here) were accurate (see Table 1 for the results of both saccade components from all subjects)

Fig. 4A-D Localization as a function of noise-burst duration. A Applied noise bursts with their associated power spectra (from top to bottom: 3 ms, 5-ms, 10-ms and 500-ms noise-burst duration, partly shown). B, C Response accuracy of subject P.H. to the $3-$ (empty circles), 5- $(+), 10-$ (stars) and 500-ms (filled circles) noise stimuli in the same format as Fig. 2. B Response azimuth; $\mathbf{C}$ response elevation; D response accuracy $(A Z$ azimuth, dashed lines; $E L$ elevation, solid lines) is quantified by the slope of the fitted regression line (together with its variance) between stimulus component and first-saccade vector component for all three subjects tested (stars J.O., empty circles P.H., filled circles J.G.). Note strong dependence of response elevation gain on stimulus duration. Such dependence is virtually absent for saccade azimuth gain
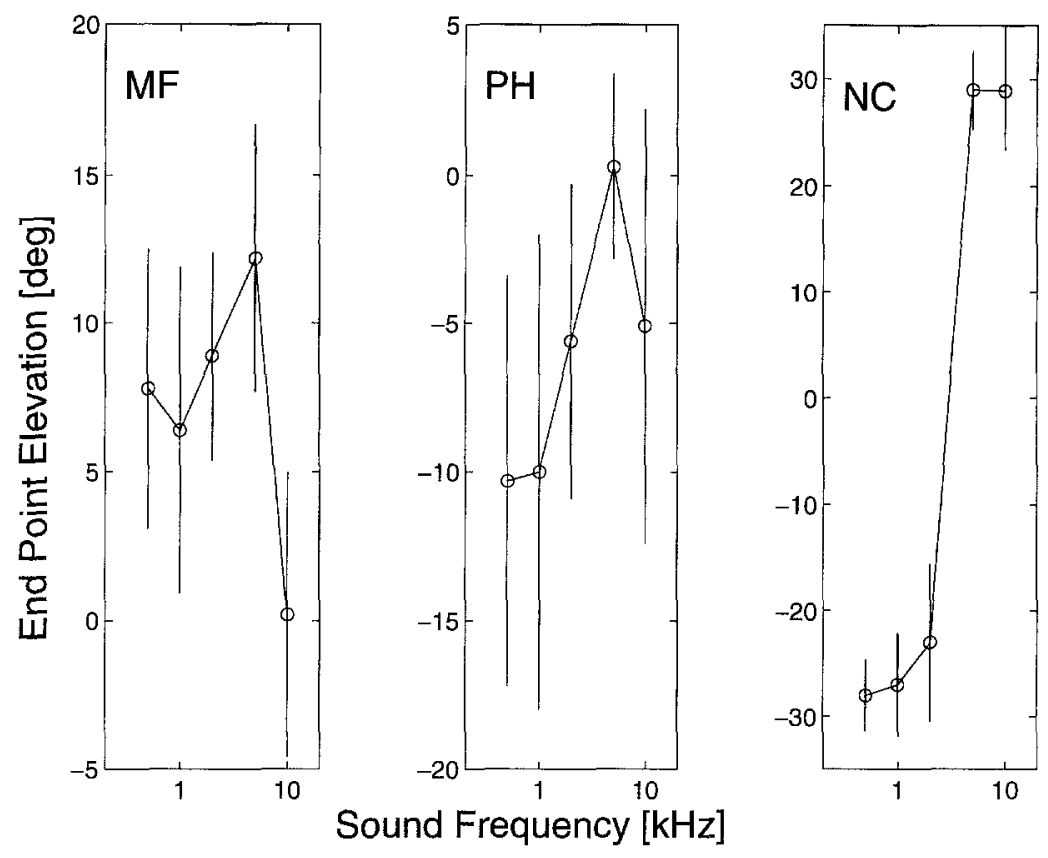
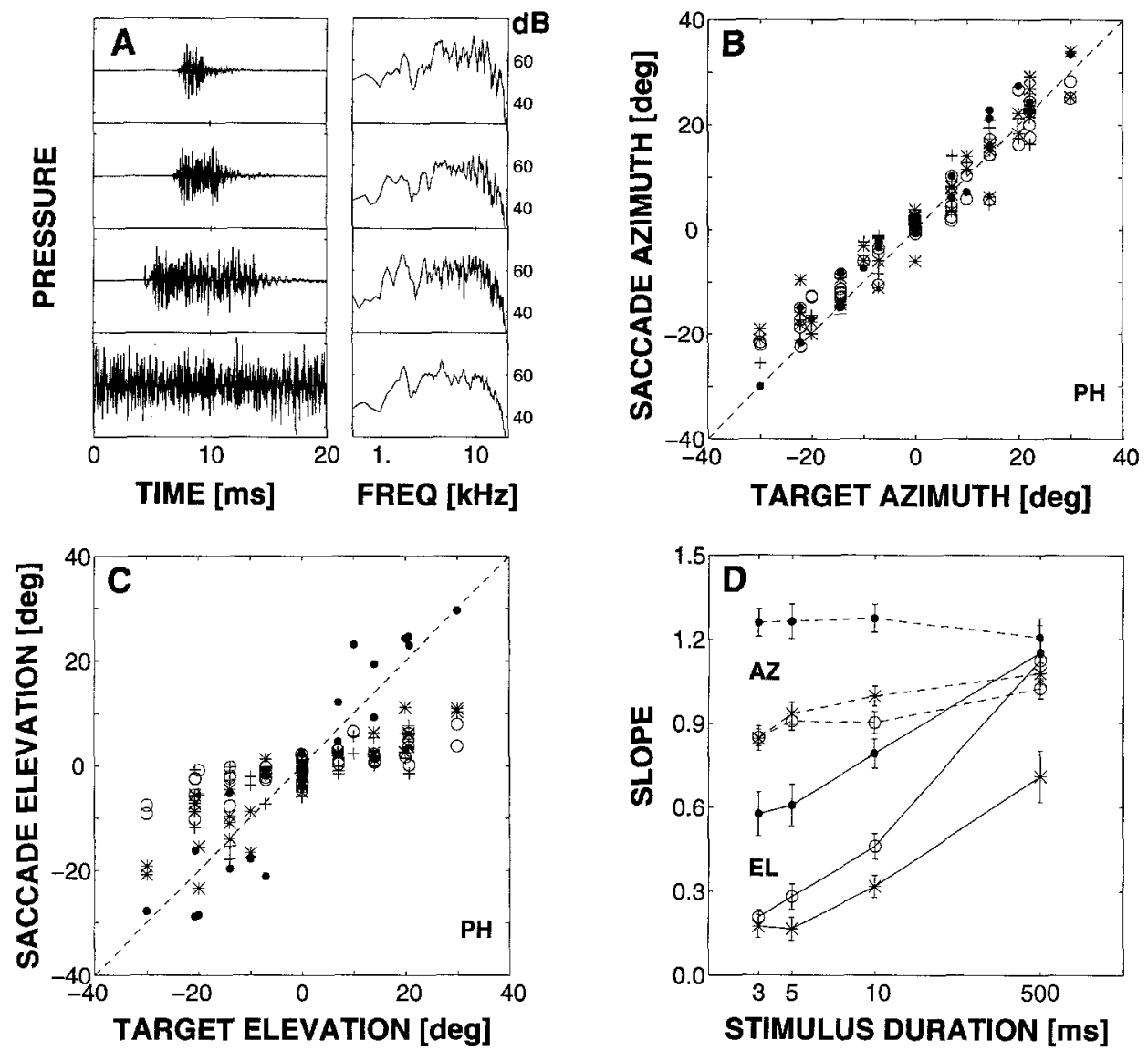

jects: the linear correlation coefficient $(r)$ between $\ln (L)$ and $R$ was always significantly different from zero $\left(P(r) \leq 10^{-3}\right)$, ranging from -0.39 to $-0.82(n \geq 65$; see Table 3 ). Interestingly, we observed that the same quantitative relation appears to hold in a manner that is roughly independent of saccade direction (see Fig. 5B). Al- though, in four of the six subjects, downward-directed auditory saccades tended to possess slightly longer latencies, the differences with upward-directed saccades were not statistically significant.

Thus, although auditory saccades may be expected to be initially programmed in Cartesian, head-centred coor- 
Table 2 Results of short-duration noise-burst experiments $(3,5$, $10,500 \mathrm{~ms}$ duration) for three subjects. All correlations are highly significant $[P(r)<0.004]$. In all three subjects, the azimuth of saccade responses was almost independent of stimulus duration. Elevation performance, however, improved strongly as stimulus dura- tion increased $[A Z, E L$ azimuth, elevation component of primary saccade responses, $a$ slope of regression line (saccade component vs component of target position), $r$ linear correlation coefficient, $n$ number of data points]

\begin{tabular}{|c|c|c|c|c|c|c|c|c|c|c|c|c|c|}
\hline \multirow[t]{2}{*}{ Subject } & \multirow[t]{2}{*}{ Comp. } & \multicolumn{3}{|l|}{$3 \mathrm{~ms}$} & \multicolumn{3}{|l|}{$5 \mathrm{~ms}$} & \multicolumn{3}{|c|}{$10 \mathrm{~ms}$} & \multicolumn{3}{|c|}{$500 \mathrm{~ms}$} \\
\hline & & $a$ & $r$ & $n$ & $a$ & $r$ & $n$ & $a$ & $r$ & $n$ & $a$ & $r$ & $n$ \\
\hline $\mathrm{PH}$ & $\begin{array}{l}\mathrm{AZ} \\
\mathrm{EL}\end{array}$ & $\begin{array}{l}0.85 \\
0.21\end{array}$ & $\begin{array}{l}0.98 \\
0.80\end{array}$ & $\begin{array}{r}41 \\
-\end{array}$ & $\begin{array}{l}0.91 \\
0.28\end{array}$ & $\begin{array}{l}0.97 \\
0.71\end{array}$ & $\begin{array}{r}44 \\
-\end{array}$ & $\begin{array}{l}0.90 \\
0.46\end{array}$ & $\begin{array}{l}0.97 \\
0.88\end{array}$ & $\begin{array}{r}44 \\
-\end{array}$ & $\begin{array}{l}1.02 \\
1.12\end{array}$ & $\begin{array}{l}0.98 \\
0.94\end{array}$ & $\begin{array}{r}24 \\
-\end{array}$ \\
\hline $\mathrm{JO}$ & $\begin{array}{l}\text { AZ } \\
\text { EL }\end{array}$ & $\begin{array}{l}0.85 \\
0.17\end{array}$ & $\begin{array}{l}0.96 \\
0.62\end{array}$ & $\begin{array}{r}48 \\
-\end{array}$ & $\begin{array}{l}0.94 \\
0.17\end{array}$ & $\begin{array}{l}0.97 \\
0.55\end{array}$ & 48 & $\begin{array}{l}1.00 \\
0.32\end{array}$ & $\begin{array}{l}0.98 \\
0.80\end{array}$ & $\begin{array}{r}47 \\
-\end{array}$ & $\begin{array}{l}1.08 \\
0.71\end{array}$ & $\begin{array}{l}0.99 \\
0.92\end{array}$ & 23 \\
\hline $\mathrm{JG}$ & $\begin{array}{l}\text { AZ } \\
\text { EL }\end{array}$ & $\begin{array}{l}1.26 \\
0.58\end{array}$ & $\begin{array}{l}0.98 \\
0.79\end{array}$ & $\begin{array}{r}48 \\
-\end{array}$ & $\begin{array}{l}1.26 \\
0.61\end{array}$ & $\begin{array}{l}0.97 \\
0.76\end{array}$ & $\begin{array}{r}48 \\
-\end{array}$ & $\begin{array}{l}1.27 \\
0.79\end{array}$ & $\begin{array}{l}0.96 \\
0.89\end{array}$ & $\begin{array}{r}48 \\
-\end{array}$ & $\begin{array}{l}1.21 \\
1.15\end{array}$ & $\begin{array}{l}0.95 \\
0.95\end{array}$ & 24 \\
\hline
\end{tabular}

Fig. 5A,B Relation between saccade vector and latency. A Latency as a function of saccade amplitude (subject J.O.), for visual saccades (stars) and auditory saccades (empty circles). Equation 5 has been fitted through both data sets ( solid line auditory, broken line visual). Note the strong decrease in latency for auditory saccades as a function of amplitude and the slight, but systematic, increase in visual saccade latencies. B Auditory saccade latency as a function of final saccade direction. In this plot only saccades with an amplitude larger than $15^{\circ}$ are shown, for which the amplitude-latency relation has reached its saturation level. No systematic dependence of saccade latency on saccade direction was obtained
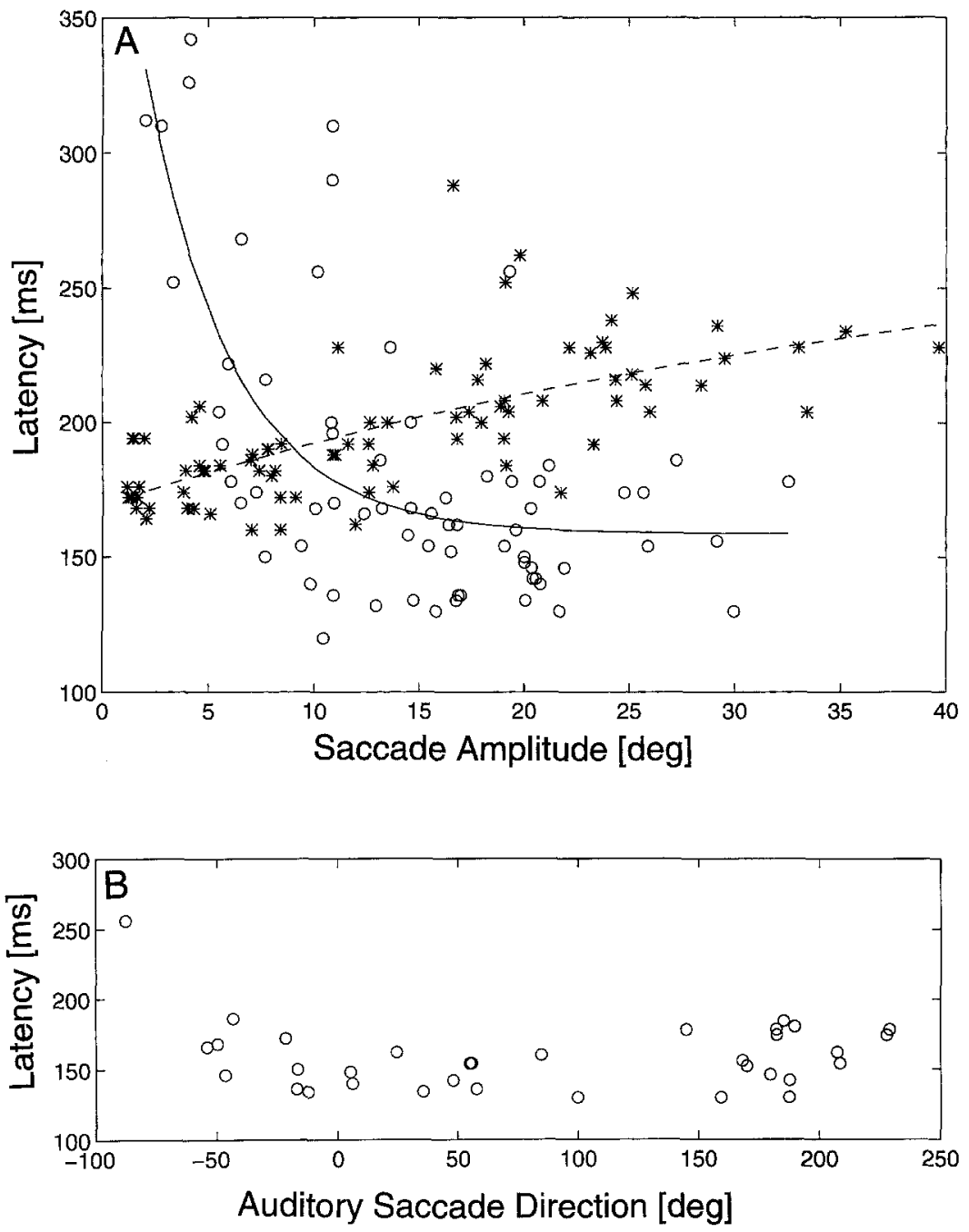

dinates (see Introduction), the execution of these saccades discloses the involvement of a system operating in a polar coordinate frame of reference. Note that although the tone-evoked saccades were not directed at the physical position of the target (see above), the same relation between saccade amplitude and latency was obtained as for broad-band noise stimuli, independent of the frequency of the tone.
By contrast, the latencies of visually evoked eye movements (Fig. 5A, asterisks, broken fit curve) increased slightly with saccade amplitude in all but two subjects [correlation between 0.59 and $0.69 ; P(r) \leq 10^{-4}$ ]. Subject P.H. diplayed no significant correlation between visual latencies and saccade amplitude $(P>0.05)$. Subject M.F. had long latencies for small saccades $\left(R<5^{\circ}\right.$; mean $240 \mathrm{~ms}$ ) and a constant latency for larger saccades (mean 
Table 3 Fit results for audidtory and visual saccade latencies (Eq. 7). $L_{0}$ is the predicted latency at $R=0^{\circ}\left(L_{0}=L_{\infty}+L_{S}\right)$. Note that the latency of auditory saccades tends to decrease with saccade amplitude $\left(L_{\infty}<L_{0}\right)$. For visual saccades the opposite trend is visible (with the exception of subject M.F.; see text). Parameter $\alpha$ measures the steepness of the relation and is of comparable magnitude for auditory saccades across subjects. For visual saccades, latencies often tended not to saturate. For those cases, $L_{\infty}$ was taken as the predicted latency value at $R=40^{\circ}$

\begin{tabular}{llllllll}
\hline \multirow{2}{*}{$\begin{array}{l}\text { Sub- } \\
\text { ject }\end{array}$} & \multicolumn{2}{l}{ Auditory } & & \multicolumn{2}{l}{ Visual } \\
\cline { 7 - 8 } \cline { 6 - 7 } & $L_{0}(\mathrm{~ms})$ & $L_{\infty}(\mathrm{ms})$ & $\alpha\left(\mathrm{deg}^{-1}\right)$ & & $L_{0}(\mathrm{~ms})$ & $L_{\mathrm{o}}(\mathrm{ms})$ & $\alpha\left(\mathrm{deg}^{-1}\right)$ \\
\hline JG & 709 & 210 & 0.26 & & 229 & 306 & - \\
JO & 440 & 159 & 0.24 & & 169 & 281 & -0.02 \\
MF & 284 & 136 & 0.18 & & 171 & 127 & 0.5 \\
NC & 560 & 303 & 0.23 & & 313 & 340 & - \\
PH & 365 & 148 & 0.12 & 188 & 206 & - \\
RW & 217 & 180 & 0.17 & 180 & 230 & - \\
\hline
\end{tabular}

$136 \mathrm{~ms})$. Though such a profile qualitatively resembles the generally obtained auditory latency distribution, visual latency saturated at a much smaller saccade amplitude for this subject.

\section{Auditory noise saccade trajectories}

As can be readily observed in Fig. 6 (right column; data from two subjects), auditory saccade trajectories evoked by broad-band noise stimuli ( $500 \mathrm{~ms}$ duration) were often considerably curved and less stereotyped than visually driven saccades (left column). Typically, the mean value of absolute curvature for auditory saccades was almost twice the value obtained for visual saccades, and

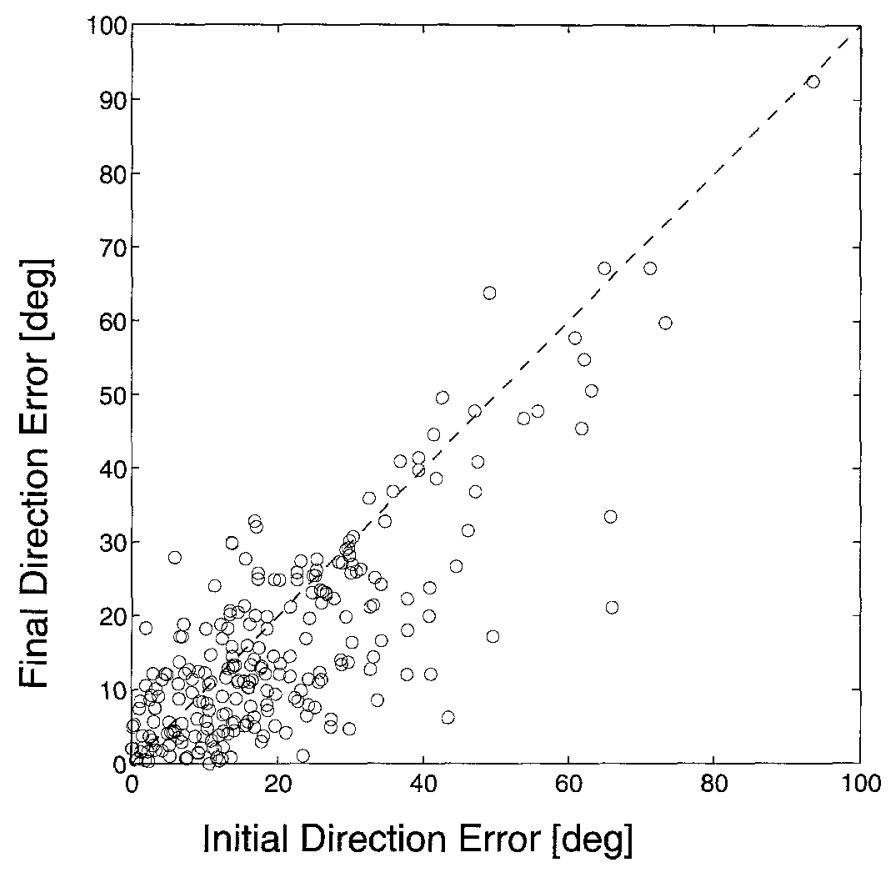

Fig. 7 Initial and final direction error of auditory saccades. This figure compares the initial error of saccade direction $\left(\Delta \Phi_{0}=\right.$ $\left.\mid \Phi_{0}-\Phi_{T}\right)$ with the direction error of the saccadic end point $(\Delta \Phi=$ $\left.\left|\Phi-\Phi_{T}\right|\right)$, pooled for all six subjects. Note that the initial error was generally larger than the final error, especially for large initial errors $\left(>20^{\circ}\right)$, where $75 \%$ of the data fall below the identity line

also the variability of curvature was much larger (see legend to Fig. 6 for details).

Closer inspection of the auditory trajectories revealed that an auditory saccade often started in a direction that was only crudely related to the physical target direction.
Fig. 6 Saccade trajectories for two subjects. Left Two-dimensional trajectories of visually guided saccades for subjects M.F. (top) and P.H. (bottom). Although most saccades are approximately straight, some movements have a considerable curvature. Absolute values of saccade curvature (see Materials and methods; mean and standard deviation): M.F., $|C|=0.05 \pm 0.04$; P.H.

$|C|=0.04 \pm 0.02 \quad(n=84)$. Right

Trajectories of saccades towards auditory broad-band noise stimuli in darkness for the same subjects and obtained in the same experimental session. Note that the auditory saccades are substantially more curved than the visual saccades, which seems in these two subjects especially pronounced for auditory targets presented in the lower oculomotor range (M.F. $|C|=0.08 \pm$ 0.06 ; P.H. $|C|=0.08 \pm 0.06$; $n=72$ )

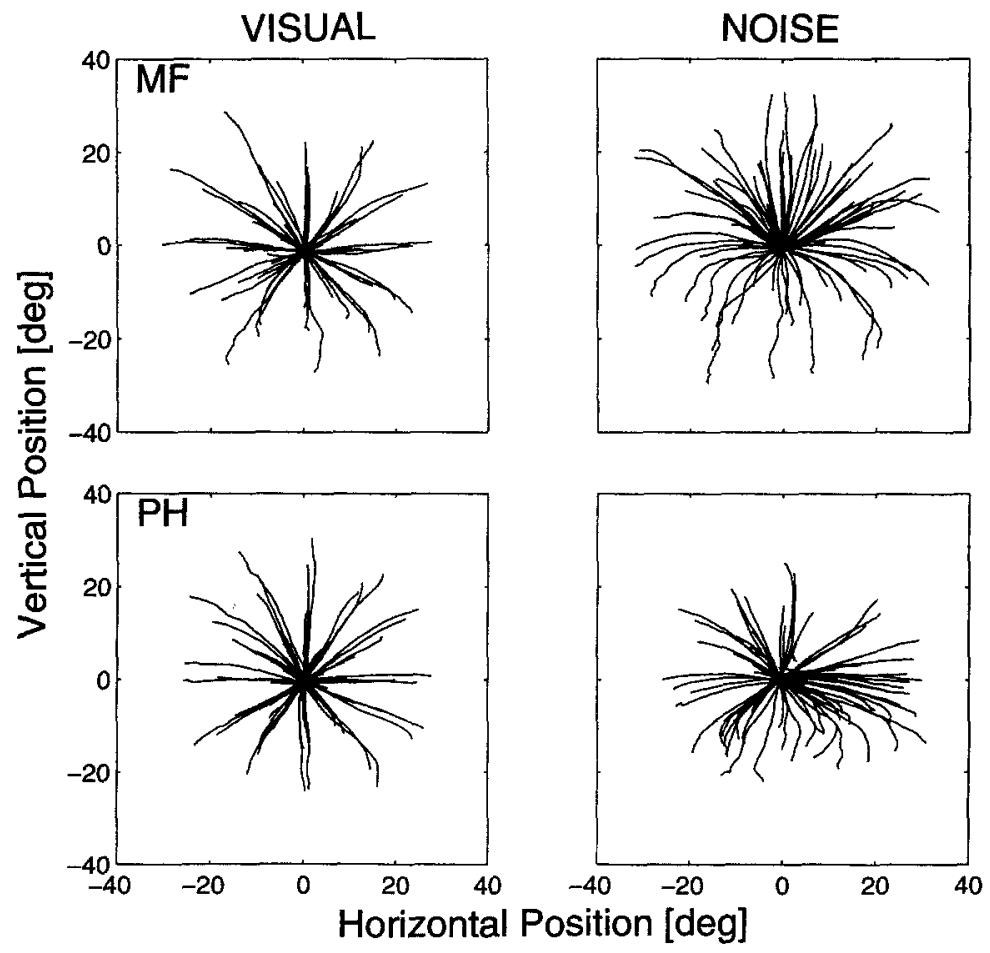




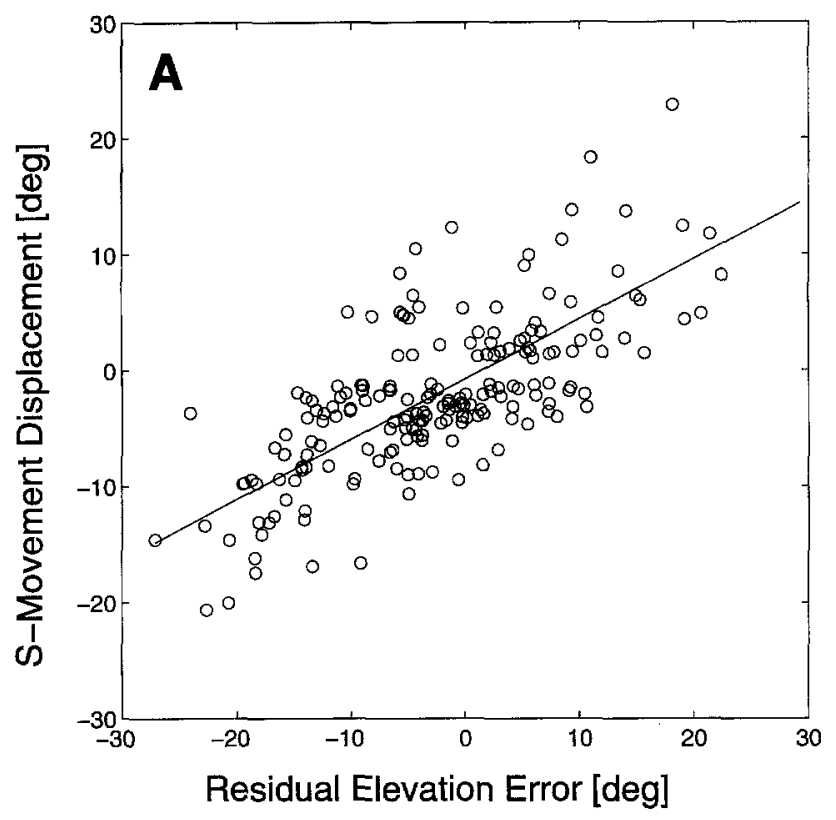

Fig. 8A,B Properties of S-movements. A Relation between residual error and S-displacement. Size and direction of the vertical Smovement as a function of the elevation error that would remain if only the P-saccade had been made. Data pooled for all six subjects $(n=278)$. S-movements with amplitudes smaller than $1^{\circ}$ were not included in the analysis (see Materials and methods). The corrective gain of the S-saccade (slope of the regression line) is 0.5 . B Distribution of the latency difference between the onsets of the Pmovement and the S-movement. Due to the assumptions in the decomposition procedure, differences are always positive. Note that the distribution has a well-defined peak at a latency difference of about $30 \mathrm{~ms}$

Subsequently, however, the movement tended to curve in mid-flight in order to guide the eye into a new direction, bringing it closer to the actual target position.

In order to quantify the corrective nature of the curvature in the saccade in a model-independent way, Fig. 7 compares the initial and final direction errors of the auditory saccades (data pooled for all subjects). Note that the initial direction error, $\Delta \Phi_{0}$, is generally larger than the final direction error. The proportion of data points falling below the identity line amounts to $66 \%$. Although this may not seem particularly impressive, this feature is especially prominent for large initial direction errors: for $\Delta \Phi_{0}>20^{\circ}, 75 \%$ of the points fall below the line. Thus, from such a first analysis, the impression was gained that the curvature in the auditory saccade trajectories was of a corrective nature.

In order to investigate this point in more depth, we applied the decomposition procedure described in Materials and methods to all auditory saccades evoked by broad-band noise. In what follows, we will describe the properties of the computed P- and S-movements, resulting from this decomposition method.

\section{Properties of $\mathrm{P}$ - and S-movements}

As may be expected from a casual inspection of Fig. 6, P-movements were made in all directions. By definition,

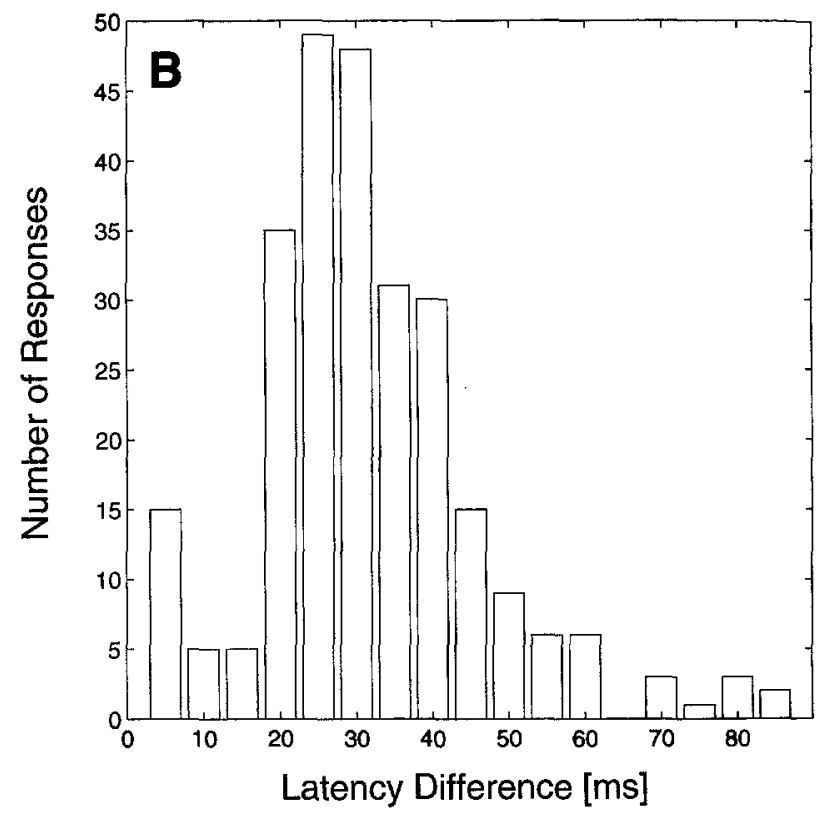

the accuracy of P-movement azimuth was equal to that of the total auditory saccade (see Materials and methods). Unlike the total movements, however, the fitted linear relationships for P-movement elevation had a slope that was substantially less than 1 (not shown). It is important to note that, nevertheless, the elevation component of the P-movements correlated significantly with target elevation. Therefore, the initial direction of the (curved) auditory saccade tended to be only roughly correct. This feature was consistently found in all subjects.

The reconstructed S-movements appeared to compensate for a substantial part of the elevation error that would remain if only the P-movement would have been made. Figure $8 \mathrm{~A}$ (pooled data from all subjects) shows that there is a highly significant relation between the residual elevation error after the P-movement and both the size and direction (indicated by its sign) of the S-movement ( $r=0.67 ; n=278$ ). The slope of the best-fit linear regression line $(a=0.5)$ indicates that on average $50 \%$ of the total error was corrected for by the S-movement.

It should be noted that, as a result of the assumptions inherent in the decomposition procedure, the onset of the $\mathrm{S}$-movement is confined to the time interval covering the duration of the P-movement (see Materials and methods). Therefore, S-movement onset and P-movement onset have to be well correlated. Nevertheless, the distribution of S-movement onsets is far from uniform within this interval, but tended to cluster at about $30 \mathrm{~ms}$ after the onset of the P-movement. This is illustrated in Fig. 8B, which shows the pooled data from our six subjects. The correlation between the onsets of the two movements that was actually obtained $(r=0.98 ; n=278)$ was significantly higher than expected for uniformly distributed S-onsets over the P-movement duration interval ( $r=0.93 \pm 0.02$, as determined by a statistical bootstrap of 100 simulations; see, e.g. Press et al. 1992). 


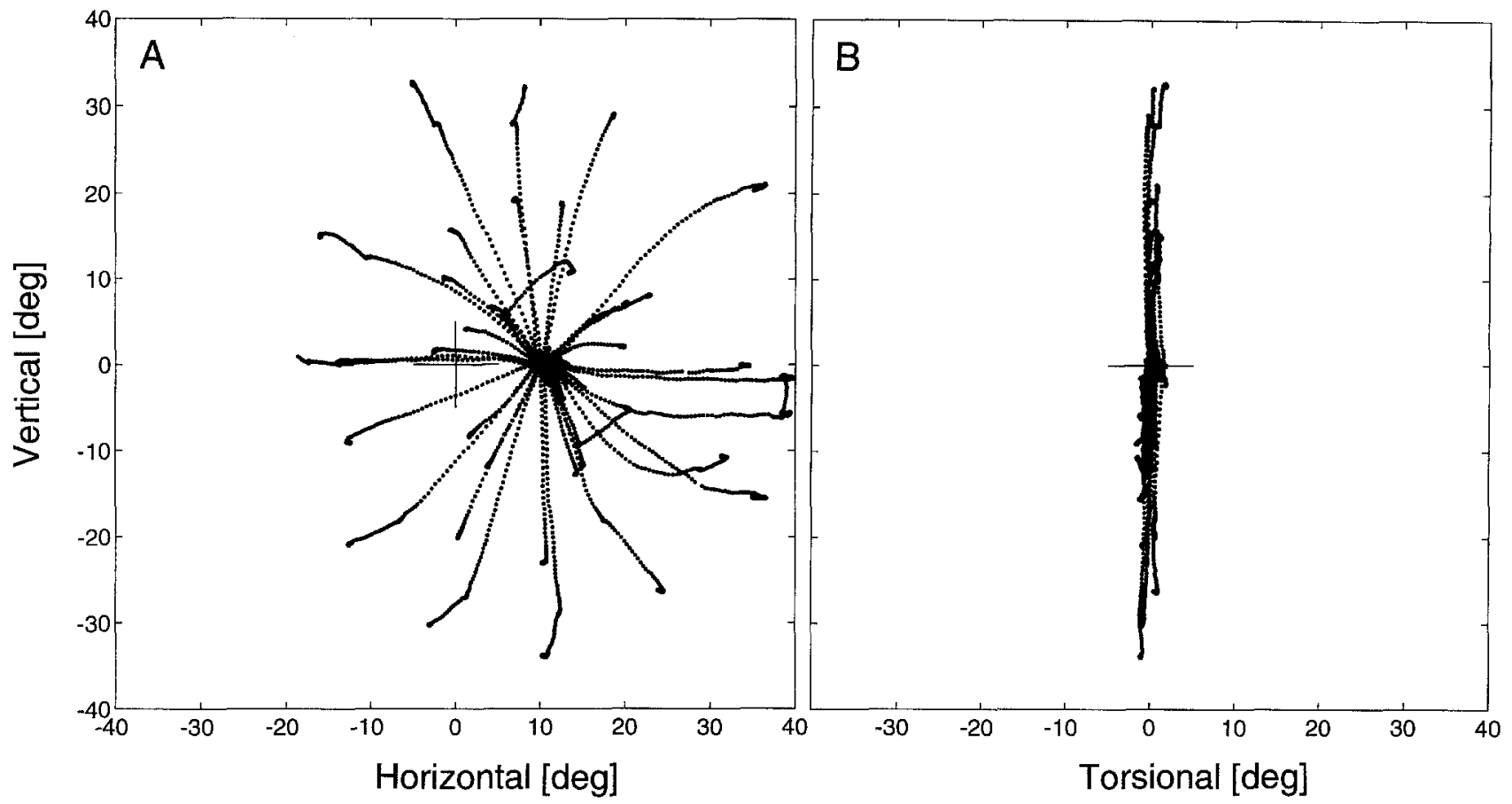

Fig. 9 Listing's law for auditory-evoked saccades. Eye positions are displayed as the three-dimensional (3D) components of a rotation axis, $\mathbf{r}=(T, H, V)^{\circ}$. This axis specifies how the eye should rotate in order to reach the current position from primary position, which is the origin $(0,0,0)$ of a right-handed, head-fixed Cartesian coordinate system (see Van Opstal 1993). The horizontal component of this rotation axis, $H$, corresponds to a vertical eye position, the vertical component of the axis, $V$, to a horizontal position. The torsional component, $T$, describes a rotation about the visual axis when the eye looks into the primary direction. A Horizontal-vertical view of the eye position rotation vectors of saccade trajectories obtained in the auditory localization experiment described in Materials and methods. Calibration cross is $\pm 5^{\circ}$. Note that the central fixation position is located at $(T, H, V) \approx(0,10,0)^{\circ}$, which is $10^{\circ}$ down from the primary position. B Torsional-vertical view of 3D eye positions. Note that data are confined to a narrow plane, centred at zero torsion (Listing's plane). Width of the plane in the torsional direction is less than $1^{\circ}\left(\sigma_{T}=0.6^{\circ}\right)$

\section{Auditory saccade kinematics in three dimensions}

It is now well established that the kinematics of visually evoked saccades in three dimensions are constrained by Listing's law. This law states that, with the head upright and at rest, the three-dimensional coordinates of the ocular rotation axis describing current eye position are confined to a plane (Listing's plane), not only while fixating but also during the eye movement (see, e.g. Tweed et al. 1990; Minken et al. 1993; Van Opstal 1993). It is still debated whether Listing's law results from a visual strategy by a requirement of keeping the orientation of the two eyes aligned for optimal binocular vision, or from a motor strategy minimizing the amount of movement about the ocular rotation axis, or whether it reflects a mechanical property of the oculomotor plant.

We have recorded eye movements in three dimensions in one subject (A.M.), in order to verify whether also the three-dimensional kinematics of auditory saccades in darkness are constrained by this law. The results are shown in Fig. 9. It is immediately clear that Listing's law is obeyed for auditory saccades with high precision, even when saccades are substantially curved.

\section{Discussion}

Accuracy of auditory saccades

In this paper we have shown that the accuracy of human auditory-evoked eye movements in two dimensions can be quite high when the sound stimulus consists of noise with a broad bandwidth. Apparently, the audio-oculomotor system has the capacity to compute the position of a sound source on the basis of binaural difference cues, as well as monaural spectral cues provided by the pinnae, and to use this result for an accurate oculomotor response.

It has been shown theoretically that, when the sound has a large bandwidth, the auditory system should be able to make the appropriate discrimination of target position in the medial plane, by assuming that the power spectrum has certain smooth properties (Zakarouskas and Cynader 1993; see also Introduction). Indeed, when a tone stimulus is used, the detection of sound elevation fails dramatically. In this case, the system has no means to relate the incoming single-frequency sound spectrum either to the intensity of the stimulus proper or to a frequency-specific filtering effect by the pinnae.

Meanwhile, the azimuth position of brief noise bursts (down to 3 ms duration; Fig. 4) could be localized just as well as in the case of longer duration broad-band noise and tone stimuli (Fig. 2, Table 1). Interestingly, despite the broad-amplitude spectra of the former two sound stimuli (see Fig. 4A), the elevation component of the 
saccadic responses was strongly affected by stimulus duration in all three subjects tested. Therefore, a broad bandwidth by itself is not sufficient to evoke an accurate localization response in two dimensions. Accurate vertical localization, which is based on spectral cues, necessitates sufficiently high spectral resolution. As a consequence of Fourier's formalism ( $\Delta f \cdot \Delta t \approx 1$ ), this computational process requires sufficient time. More experiments are needed in order to assess the precise time course of the vertical localization percept. On the basis of our first results (Table 2) a preliminary estimate would indicate a value exceeding $10 \mathrm{~ms}$.

Interestingly, when the auditory system has no means to derive the elevation of a sound, the vertical component of the saccadic response is not random. Rather, subjects seem to choose a default elevation (Figs. 2C, 3), which depends consistently on the frequency of the sound. We observed that the relation between sound frequency and evoked elevation response is also subject-specific. This observation is in line with psychophysical data suggesting that the specific shape of such a relationship, expressed by the subject's performance in an auditory localization task, may be due to the idiosyncratic filtering properties of the pinnae (Rogers and Butler 1992; Butler and Musicant 1993).

In this respect it may be of relevance that the initial movement direction to noise stimuli is, despite its inaccuracy, well correlated with target direction and should therefore not be regarded as a mere default response, as in the case of tone stimuli. This could fit nicely with the idea, strongly supported by the brief-duration noise-burst data, that the high spectral resolution required for a better localization cannot be obtained within brief processing times.

Our eye movement data indicate that both the two-dimensional percept of auditory target position and the subsequent programming of an accurate orienting movement are complete after approximately $200 \mathrm{~ms}$. Our model-free analysis (Fig. 7) and our reconstruction method (Fig. 8A; see also below) both suggest that the accuracy of the acoustically evoked orienting movement may indeed improve over time: after a mean latency of about $150 \mathrm{~ms}$ (see, e.g. Fig. 5) a first estimate of the acoustic target position becomes apparent in the initial direction of the eye movement. Then, after a fixed time interval of approximately $30 \mathrm{~ms}$ (Fig. 8B), the change in direction of the movement suggests that target position is specified more precisely (Fig. 7).

A similar suggestion was made by Jay and Sparks (1990), who noted that the auditory response was often broken up into a number of smaller saccades, which brought the eye in successive steps closer to the target. Although these authors report that $20 \%$ of their recorded auditory responses displayed this "staircase" pattern, such responses were quite rare in our own data base.

It should further be noted that such detailed information about the auditory localization process would be difficult to obtain by psychophysical procedures which confine the subject's responses to a limited array of possible target positions, or in which the kinematics and accuracy of the response are not available.

\section{Latency}

We confirm and extend previous findings (Zahn et al. 1979; Jay and Sparks 1990) that the latency of auditory evoked eye movements decreases with increasing saccade amplitude. By contrast, latencies of visually driven saccades are fairly constant over a large amplitude range. In most subjects, only a small (but significant) increase in response latency with target eccentricity was obtained. It is not clear why auditory latencies are differently related to saccade amplitude than visual latencies.

A new additional finding in this study is that the amplitude-latency dependence seems to be independent of saccade direction and hence occurs in a radial-symmetric way. We have checked, by replotting the auditory latency data of Jay and Sparks (1990), that their data too appear to reflect this radial symmetry. As was outlined in the Introduction, auditory localization is initially confined to a Cartesian, craniocentric frame of reference, due to the early separation of the horizontal and vertical localization cues. It is therefore of interest that the studies by Zahn et al. (1979) and by Jay and Sparks (1990) both suggest that auditory saccade latency is related to the oculocentric coordinates of an auditory target rather than to the acoustic head-centred eccentricity. Our latency data further support this hypothesis.

Auditory saccades are thought to be represented in the same oculocentric format as visual saccades at the level of the deep layers of the SC (Jay and Sparks 1987). Thus, the rotation-symmetric and oculocentric properties of auditory saccade latencies could be the result of a collicular involvement in auditory saccade initiation.

This hypothesis is further supported by recent findings from our group which indicate that the initiation of visually evoked saccades is influenced by the presence of an auditory target. The effect could be explained by a spatial-temporal interaction at a neural stage, where both the visual and the auditory target are programmed in the same spatial format, such as has been found in the motor map of the SC (Lueck et al. 1990; Frens et al. 1995).

\section{Auditory and visual saccade trajectories}

We observed substantial differences between the trajectories of visually and acoustically driven saccades. Most notably, the trajectories of saccades towards broad-band noise targets were generally more curved than visual saccades (Fig. 6). Interestingly, the curvature of auditory saccades was found to be corrective, since it tended to improve the accuracy of the direction of the saccade (Fig. 7).

Note that this correction cannot be the result of auditory feedback, since the head position of our subjects remained fixed throughout the experiment. Nor may the 
observed curvature be explained by a property of the oculomotor plant, because the trajectories of the visually evoked eye movements were in general quite different. We also believe that the observed curvature of auditory saccades is not due to the lack of visual stimulation: in a recent study it has been shown that memory-guided saccades in the dark and visually evoked saccades display both qualitatively and quantitatively similar saccade trajectories (Smit et al. 1990).

In short, our auditory saccade data suggest that the corrective curvature within the movement may reflect ongoing signal processing related to the auditory localization process.

\section{Models of curved saccades}

It has been shown in earlier studies that strongly curved saccades may also be evoked by visual stimuli, e.g. in a situation where the initial visual target rapidly changes direction in a double-step task (Ottes et al. 1982; Findlay and Harris 1984; Van Gisbergen et al. 1987; Minken et al. 1993). It has recently been noted by Minken et al. (1993) that such strong curvature is incompatible with the notion of a single-axis ("shortest path") ocular rotation.

Different models may explain such curved trajectories (see Ottes et al. 1982; Van Gisbergen et al. 1987; Minken et al. 1993). For example, a two-dimensional extension of the well-known internal feedback model of Robinson (1975) assumes that the brainstem saccade generator is driven by a motor error signal which is continuously updated by newly arriving target information (e.g. Ottes et al. 1982). In such a feedback model, motor error is continuously computed by subtracting current eye position from the desired target position in the head.

Alternatively, curved saccades may be envisaged as the result of a superposition of two preprogrammed movements, elicited by the two stimulus steps (Ottes et al. 1982). On the basis of a quantitative analysis, it was observed by Van Gisbergen et al. (1987) that curved double-step saccades may be equally well described by both models.

In the present paper, we have not attempted to resolve this feedforward versus feedback dichotomy, since on the basis of behavioural data alone this may be next to impossible. However, we propose that also the generation of auditory-evoked saccades may be subject to a continuous updating mechanism. Thus, also in this case, the curved auditory saccade may be due to: (1) either a continuously changing motor error signal, resulting from new information concerning the head-centred auditory target position; (2) or to the superposition of two feedforward-programmed saccades. In the latter scheme, the second saccade must take into account the oculocentric coordinates of the updated target position, as well as the estimated motor error after the first saccade.

\section{Decomposition procedure}

As has been outlined in Materials and methods, the decomposition is not unique and therefore has to be subjected to constraints. This problem is also encountered in the analysis of curved visual, double-step responses, despite the clear lay-out of the target configuration.

The starting point of our decomposition analysis has been the assumption that the observed responses reflect a property akin to the audio-oculomotor system. Indeed, the strong and variable curvature in the auditory saccades was not observed in visually evoked responses to single targets. Although also saccades to single visual targets and memory-guided saccades tend to be slightly curved, the curvature is usually quite systematic and reproducible (Smit et al. 1990; see also Fig. 6). Although it cannot be excluded that an updating mechanism resembling that proposed for the double-step saccades or the auditory saccades may also underlie the generation of saccades to single visual targets, the strong reproducibility of the latter suggests a different, perhaps even a hardwired, mechanism.

Inspired by the notion that initially the process of auditory localization involves independent channels for the extraction of target azimuth and target elevation (see Introduction), we attempted in a previous study to describe an auditory saccade as the superposition of a purely horizontal and a purely vertical saccadic movement (Frens and Van Opstal 1994a). Although often the auditory saccades seemed to start in a horizontal direction, giving support to this notion (see, e.g. Fig. 6, for saccades to auditory targets in the lower oculomotor field of subjects M.F. and P.H.), this feature was not consistent throughout the entire two-dimensional oculomotor range (cf. the saccades with an upward component in Fig. 6). Moreover, in some subjects the deviations from a simple horizontal/vertical segregation of saccade trajectories was more prominent than in other subjects.

In short, the finding that horizontal/vertical components of auditory saccades starting in oblique directions display a kinematic component cross-coupling ("stretching") similar to that found for visually evoked saccades (Van Gisbergen et al. 1985; see also Introduction) is at odds with the hypothesis that auditory saccades may be treated as a superposition of saccades generated by independent azimuth/elevation channels.

The reconstruction procedure applied in the present paper therefore assumes that the response made by the audio-oculomotor system may be described as a superposition of two overlapping movements, of which the initial direction is not necessarily horizontal. Note that this reconstruction procedure is compatible with both models described above. Based on our localization data (discussed above) we conjecture that the first command (resulting in the P-movement) is predominantly based on binaural signal processing and is already accurate in as far as target azimuth is concerned. However, due to a first rough spectral analysis on the acoustic signal, an initial estimate of target elevation can nevertheless be 
Fig. 10 Main sequence relations of visual and auditory saccades. $R-V_{\max }$ relations of visual saccades $(+)$, reconstructed P-movements (empty circles) and $\mathrm{S}$-movements (stars) for four subjects (J.G., J.O., M.F., P.H.). Note that all three movement types follow approximately similar relationships (see also Table 4 for quantitative details)

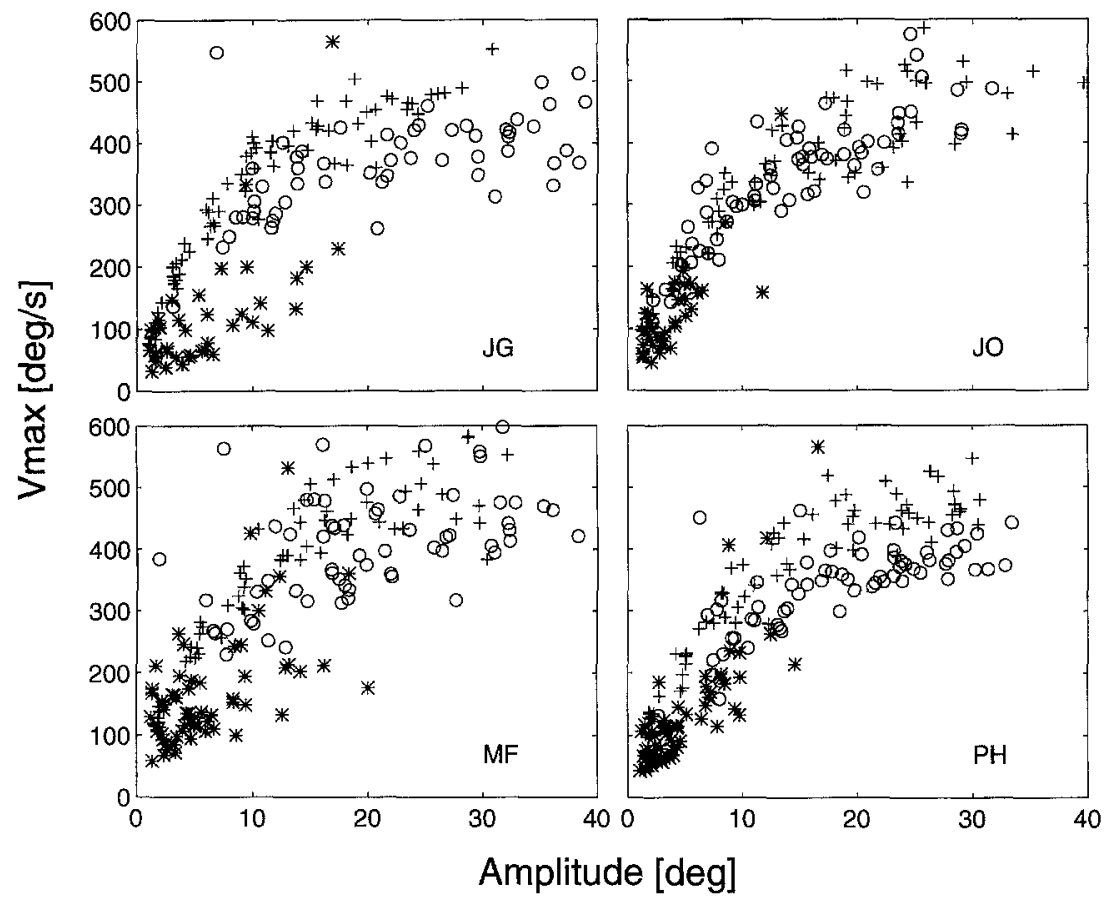

Table 4 Peak velocity saturation levels for visual saccades and auditory P-movements (pooled over directions) determined for saccade amplitudes exceeding $20^{\circ}$. Although visual saccades tend to be somewhat faster for all subjects, differences in the means are highly significant in only two of six subjects (Student's $t$-test). The right-hand side provides the slopes of the fitted linear regression lines for the visual saccades and P- and S-saccades with amplitudes smaller than $20^{\circ}$ (pooled directions). Note that $V$ - and P-saccades are equally fast, whereas $\mathrm{S}$-saccades are systematically slower

\begin{tabular}{|c|c|c|c|c|c|c|}
\hline Subject & \multicolumn{3}{|c|}{ Saturation level main sequence } & \multicolumn{3}{|c|}{ Slope $R<20^{\circ}$} \\
\hline $\mathrm{JG}$ & $462 \pm 43$ & $397 \pm 52$ & 0.0002 & 43 & 38 & 25 \\
\hline $\mathrm{JO}$ & $459 \pm 68$ & $437 \pm 66$ & 0.32 & 39 & 37 & 31 \\
\hline MF & $513 \pm 70$ & $459 \pm 90$ & 0.03 & 40 & 43 & 27 \\
\hline RW & $398 \pm 51$ & $362 \pm 95$ & 0.26 & 39 & 43 & 23 \\
\hline
\end{tabular}

made (see also above). The second command (giving rise to the S-movement), which on average affects the initial response with a delay of about $30 \mathrm{~ms}$ (see Fig. 8B), is hypothesized to result from a further analysis of the spectral cues. Depending on the underlying model (see above), the second command may also have to take the coordinates of the P-movement into account.

\section{Saccade kinematics}

An interesting emerging result from the proposed decomposition procedure is that both the reconstructed $\mathrm{P}$ movement and the S-movement turn out to be of a saccadic nature. A comparison between these straight hypothetical auditory-evoked movements and visually guided saccades (Fig. 10) shows that all three movement types obey similar main sequence relations. Note that this fea- ture is not trivial: particularly the S-movement could have resembled a very slow movement, or even a mere irregular oscillation around the initial saccade direction. Indeed, the fastest movements of all three types turned out to be indistinguishable. This is illustrated in Fig. 10 for four of our subjects (see Table 4 for the quantitative results of all subjects).

This is in striking contrast to previous claims in the literature (Zahn et al. 1979; Zambarbieri et al. 1982), suggesting that auditory saccades are considerably slower than visually evoked eye movements. A possible explanation for this discrepancy could be that, by measuring only the horizontal component of auditory saccades, peak velocities may systematically have been underestimated by a factor of $1 / \cos \left(\Phi_{0}\right)$. Especially for tone stimuli, the real initial direction of the saccade may be quite different from $\Phi_{0}=0^{\circ}$ (see, e.g. subject N.C. in Fig. 3 as an extreme example). 


\section{Three-dimensional rotational kinematics}

We showed that Listing's law is accurately obeyed also for auditory saccades in darkness (Fig. 9). These data therefore argue against a purely visual basis for the functional role of Listing's law. The law is also obeyed when the auditory saccades are substantially curved. Therefore, in line with the observation of Minken et al. (1993) on visually evoked curved eye movements, it seems likely that also the auditory-evoked oculomotor commands express the required changes for the ocular rotation axis in two-dimensional, rather than in three-dimensional, coordinates. Clearly, electrophysiological recordings in neural structures known to be heavily involved in saccade generation, such as the midbrain (SC) and brainstem saccade-burst generator, during strongly curved saccadic responses are needed in order to discriminate among the various models.

Acknowledgements This work was supported by the University of Nijmegen and the Mucom II program (6615) of the European ESPRIT initiative. We greatly acknowledge the technical assistance of $\mathrm{H}$. Kleijnen and T. Van Dreumel. We also thank Dr. A. Minken for his involvement in the three-dimensional recordings, and Drs. J. Goossens and P. Hofman for participating in the shortduration burst experiments. Finally, we would like to express our gratitude to both anonymous referees, whose detailed comments led to substantial improvement of our paper.

\section{References}

Bahill AT, Clark MR, Stark L (1975) The main sequence: a tool for studying human eye movements. Math Biosci 24: 191 204

Butler RA, Musicant AD (1993) Binaural localization: influence of stimulus frequency and the linkage to covert peak areas. Hear Res 67: 220-229

Clarey JC, Barone P, Imig TJ (1992) Physiology of thalamus and cortex. In: Popper AN, Fay RR (eds) The mammalian auditory pathway: neurophysiology. Springer, Berlin Heidelberg New York

Collewijn H, Van der Mark F, Jansen TJ (1975) Precise recording of human eye movements. Vision Res 15: 447-450

Collewijn H, Van der Steen J, Ferman L, Jansen TC (1985) Human ocular counterroll: assessment of static and dynamic properties from electromagnetic scleral coil recordings. Exp Brain Res 59: 185-196

Findlay JM, Harris LR (1984) Small saccades to double-stepped targets moving in two dimensions. In: Gale AG, Johnson F (eds) Theoretical and applied aspects of eye movement research. Elsevier, Amsterdam, pp 71-78

Frens MA, Van Opstal AJ (1994a) Auditory-evoked saccades in two dimensions: dynamical characteristics, influence of eye position, and sound source spectrum. In: Delgado-García J, Godaux E, Vidal PP (eds) Neural mechanisms underlying gaze control. Pergamon, Oxford, pp 329-339

Frens MA, Van Opstal AJ (1994b) Dynamical properties of auditory-evoked saccades. Neurosci Abstr 20: 1402

Frens MA, Van Opstal AJ, Van der Willigen RW (1995) Spatial and temporal factors determine audio-visual interactions in human saccadic eye movements. Percept Psychophys 57:802816

Hess BJM, Van Opstal AJ, Straumann D, Hepp K (1992) Calibration of three-dimensional eye positions using search coil signals in the rhesus monkey. Vision Res 32: 1647-1654
Irvine DRF (1992) Physiology of the auditory brainstem. In: Popper AN, Fay RR (eds) The mammalian auditory pathway: neurophysiology. Springer, Berlin Heidelberg New York

Jay MF, Sparks DL (1987) Sensorimotor integration in the primate superior colliculus. I. Motor convergence. J Neurophysiol 57: 22-34

Jay MF, Sparks DL (1990) Localization of auditory and visual targets for the initiation of saccadic eye movements. In: Berkley MA, Stebbins WC (eds) Basic mechanisms. (Comparative perception, vol I) Wiley, New York

Kalesnykas RP, Hallett PE (1994) Retinal eccentricity and the latency of saccades. Vision Res 34: 517-531

Kistler DJ, Wightman FL (1992) A model of head-related transfer functions based on principal component analysis and minimum-phase reconstruction. J Acoust Soc Am 3: 1637-1647

Lueck CJ, Crawford TJ, Savage CJ, Kennard C (1990) Auditoryvisual interaction in the generation of saccades in man. Exp Brain Res 82: 149-157

Middlebrooks JC, Green DM (1991) Sound localization by human listeners. Ann Rev Psychol 42: 135-159

Minken AWH, Van Opstal AJ, Van Gisbergen JAM (1993) Threedimensional analysis of strongly curved saccades elicited by double-step stimuli. Exp Brain Res 93: 521-533

Ottes FP, Van Gisbergen JA.M., Eggermont JJ (1982) Responses of the saccadic system to sudden changes in target direction. In: Roucoux A, Crommelinck M (eds) Physiological and pathological aspects of eye movements. Junk, The Hague, pp 313-318

Press WH, Flannery BP, Teukolsky SA, Vettering WT (1992) Numerical recipes in C, 2nd edn. Cambridge University Press, Cambridge

Robinson DA (1975) Oculomotor control signals. In: Lennerstrand G, Bach-y-Rita P (eds) Basic mechanisms of ocular motility and their clinical implications. Pergamon, Oxford, pp 337-374

Rogers ME, Butler AE (1992) The linkage between stimulus frequency and covert peak areas as it relates to monaural localization. Percept Psychophys 52: 536-546

Rumelhart DE, Hinton GE, Williams RJ (1986) Learning internal representations by error propagation. In: Rumelhart DE, McClelland JL (eds) Parallel distributed processing: explorations in the microstructure of cognition, vol I. MTT Press, Cambridge, Mass, pp $318-362$

Smit AC, Van Opstal AJ, Van Gisbergen JAM (1990) Component stretching in fast and slow oblique saccades in the human. Exp Brain Res 81: 325-334

Tweed D, Cadera W, Vilis T (1990) Computing three-dimensional eye position quaternions and eye velocity from search coil signals. Vision Res 30: 97-110

Van Gisbergen JAM, Van Opstal AJ, Schoenmakers JJM (1985) Experimental test for the generation of oblique saccades. Exp Brain Res 57: 321-336

Van Gisbergen JAM, Van Opstal AJ, Roebroek JGH (1987) Stimulus-induced midflight modification of saccade trajectories. In: O'Regan JK, Lévy-Schoen A (eds) Eye movements: from physiology to cognition. Elsevier, Amsterdam, pp 27-36

Van Opstal AJ (1993) Representation of eye position in three dimensions. In: Berthoz A (ed) Multisensory control of movement. Oxford University Press, Oxford, pp 27-41

Whittington DA, Hepp-Reymond MC, Flood W (1981) Eye and head movements to auditory targets. Exp Brain Res 41: $385-363$

Zahn JR, Abel LA, Dell'Osso LF, Daroff RB (1979) The audioocular response: intersensory delay. Sens Proc 3: 60-65

Zakarouskas P, Cynader MS (1993) A computational theory of spectral cue localization. J Acoust Soc Am 94: 1323-1331

Zambarbieri D, Schmid R, Prablanc C, Magenes G (1981) Characteristics of eye movements evoked by the presentation of acoustic targets. In: Fuchs AF, Becker W (eds) Progress in oculomotor research. Elsevier/North-Holland, Amsterdam, pp $559-566$

Zambarbieri D, Schmid R, Magenes G, Prablanc C (1982) Saccadic responses evoked by presentation of visual and auditory targets. Exp Brain Res 47: 417-427 